\title{
Hyperlipidemia in Coronary Heart Disease
}

\author{
III. EVALUATION OF LIPOPROTEIN PHENOTYPES OF 156 \\ GENETICALLY DEFINED SURVIVORS OF \\ MYOCARDIAL INFARCTION
}

\author{
Wimliam R. Hazzard, Joseph L. Goldstein, Helmut G. Schrott, \\ ARNo G. MotulsKy, and Edwin L. BiERMan with the technical assistance of \\ Margaret R. Poole, Ellen D. Campbell, and Mary Jo Levinski
}

From the Departments of Medicine (Division of Metabolism and Gerontology, Veterans Administration Hospital, and Division of Medical Genetics, University Hospital) and Genetics, University of Washington, Seattle, Washington 98195

A B S T R A C T Although analysis of lipoprotein phenotypes is widely used to diagnose and classify the familial hyperlipidemias, an evaluation of this system as a method for genetic classification has hitherto not been published. The present study of 156 genetically defined survivors of myocardial infarction was therefore designed to examine the relationship between lipoprotein phenotypes and genetic lipid disorders. The lipoprotein phenotype of each survivor was determined primarily by measurement of his plasma triglyceride and low density lipoprotein (LDL)-cholesterol concentrations; his genetic disorder was identified by analysis of whole plasma cholesterol and triglyceride levels in relatives.

The mean levels of LDL-cholesterol discriminated statistically among the three monogenic lipid disorders: it was highest in survivors with familial hypercholestero-

This work was presented in part at the 85th Session of the Association of American Physicians, Atlantic City, N. J., 2-3 May 1972, and published in the Transactions (1).

Dr. Goldstein was supported by Special National Institutes of Health Fellowship GM 4784-01 and is now a Research Career Development Awardee (1-K4-GM 70, 277-01) from the National Institute of General Medical Sciences. His present address is the Division of Medical Genetics, Department of Internal Medicine, University of Texas Southwestern Medical School, Dallas, Tex. 75235. Dr. Hazzard was the recipient of a Clinical Investigatorship of the Veterans Administration and is now an Investigator of the Howard Hughes Medical Institute. Dr. Schrott was supported by Special National Institutes of Health Fellowship HE-48 695. His present address is the Mayo Clinic and Mayo Foundation, Rochester, Minn. 55901.

Received for publication 20 September 1972 and in revised form 19 February 1973. lemia $(261 \pm 61 \mathrm{mg} / 100 \mathrm{ml}$ [mean $\pm \mathrm{SD}])$; intermediate in those with familial combined hyperlipidemia (197士 $50)$; and lowest in those with familial hypertriglyceridemia $(155 \pm 36)(P<0.005$ among the three groups). However, on an individual basis no lipoprotein pattern proved to be specific for any particular genetic lipid disorder; conversely, no genetic disorder was specified by a single lipoprotein pattern. This lack of correlation occurred for the following reasons: $(a)$ individual LDLcholesterol levels frequently overlapped between disorders; $(b)$ in many instances a small quantitative change in the level of either LDL-cholesterol or whole plasma triglyceride caused qualitative differences in lipoprotein phenotypes, especially in individuals with familial combined hyperlipidemia, who showed variable expression (types II $a, \mathrm{II} b, \mathrm{IV}$, or $\mathrm{V}$ ) ; (c) lipoprotein phenotypes failed to distinguish among monogenic, polygenic, and sporadic forms of hyperlipidemia; $(d)$ clofibrate treatment of some survivors with genetic forms of hyperlipidemia caused their levels of triglyceride and LDL-cholesterol to fall below the 95th percentile, thus resulting in a normal phenotype; and $(e) \beta$-migrating very low density lipoproteins $(\beta-\mathrm{VLDL})$, previously considered a specific marker for the type III hyperlipidemic disorder, was identified in several survivors with different lipoprotein characteristics and familial lipid distributions.

These studies indicate that lipoprotein phenotypes are not qualitative markers in the genetic sense but instead are quantitative parameters which may vary among different individuals with the same genetic lipid disorder. It would therefore seem likely that a genetic classification of the individual hyperlipidemic patient with coronary 
heart disease made from a quantitative analysis of lipid levels in his relatives may provide a more meaningful approach than determination of lipoprotein phenotypes.

\section{INTRODUCTION}

As knowledge of the metabolism of lipids and lipoproteins has advanced, several methods for classifying the hyperlipidemias have been proposed $(2-6)$. The system of classification in widest current use-analysis of lipoprotein phenotypes-was originally developed by Fredrickson, Levy, and Lees (5) and has since been modified and endorsed by the World Health Organization (7). In its present form, this system is based on the level of cholesterol in low density lipoproteins (LDL), ${ }^{1}$ the concentration of triglyceride in whole plasma, the electrophoretic mobility of very low density lipoproteins (VLDL), and the presence or absence of chylomicrons in plasma after an overnight fast (7). Six abnormal lipoprotein patterns (types I, II $a, \mathrm{II} b, \mathrm{III}, \mathrm{IV}$, and V) are presently recognized. The relationship of each to different genetically defined hyperlipidemic disorders has not yet been determined except for the familial type I pattern, which results from an autosomal recessive disorder of lipoprotein lipase deficiency (8). It has been generally assumed, however, that the presence of the type II $a$ or II $b$ pattern in several members of the same family denotes the genetic disorder, familial hypercholesterolemia; that a familial type IV pattern indicates the disorder, familial hypertriglyceridemia; and that the type III and the type $\mathrm{V}$ patterns when occurring in familial form each indicate distinct and separate genetic disorders $(5,9)$.

To test the validity of these assumptions, lipoprotein phenotypes were determined in 129 hyperlipidemic and 27 normolipidemic survivors of myocardial infarction. The phenotype of each survivor was compared with his genetic diagnosis, which had been independently made on the basis of plasma lipid levels in his relatives using the approach described in the accompanying paper (10). The data from this study have also provided useful information regarding: $(a)$ the variability of lipid levels of survivors of myocardial infarction on repeat testing, $(b)$ diagnostic value of a simple whole plasma cholesterol measurement in reflecting its concentration in LDL, and (c) the frequency of the type III lipoprotein pattern among consecutively studied survivors of myocardial infarction.

\section{METHODS}

Selection of survivors for analysis of lipoprotein phenotypes. Lipoprotein phenotypes were determined on repeat

${ }^{1}$ Abbreviations used in this paper: LDL, low density lipoproteins; VLDL, very low density lipoproteins; HDL, high density lipoproteins; PVP, polyvinylpyrollidone. fasting plasma specimens collected from 156 of the 176 survivors who underwent family studies (10) on the basis of an initial lipid analysis 3 mo after their myocardial infarction (11). The mean interval between initial and repeat samplings was 117 days. Of the 20 survivors who were not retested, 5 had died in the interim, 5 left the Seattle area, 1 suffered a recurrent myocardial infarction, 5 could not be located, and 4 refused to participate. The methods for collection of the repeat fasting blood sample and for the separation of plasma were identical with those used for the initial sample (11).

Laboratory procedures. To prevent changes in the lipid composition (12) and electrophoretic patterns (5) of lipoproteins which occur upon prolonged standing of plasma at ambient temperature, all plasma specimens were centrifuged in the Spinco L2-65B preparative ultracentrifuge (Beckman Instruments, Inc., Spinco Div., Palo Alto, Calif.) within $6 \mathrm{~h}$ after blood collection. For this procedure $6.5 \mathrm{ml}$ of plasma was overlayered with an equal volume of $d 1.006$ saline and centrifuged overnight $(>12 \mathrm{~h})$ at $48,000 \mathrm{rpm}$ in the angle $50 \mathrm{Ti}$ rotor at $23^{\circ}$ so as to float all $d<1.006$ lipoproteins (VLDL) into the upper half of the tube. The tube was then sliced in the middle, and the supernatant $\left(S_{\mathbf{r}}>20\right.$, VLDL) and infranatant ( $S_{\boldsymbol{t}}<20, \mathrm{LDL}$ plus $\left.\mathrm{HDL}\right)$ fractions were collected quantitatively. Each fraction and a portion of whole plasma were analyzed for cholesterol and triglyceride content by methods identical to those employed for the initial plasma sample (11). The concentration of cholesterol in HDL was determined by heparin-manganese precipitation of whole plasma (13). The cholesterol content of LDL was calculated as its concentration in whole plasma less the sum of that in VLDL plus HDL (9).

Lipoprotein electrophoresis of whole plasma was performed in both agarose (14) and polyacrylamide gels (15). The supernatant $\left(S_{f}>20\right)$ and infranatant $\left(S_{t}<20\right)$ fractions were also subjected to electrophoresis in agarose gel. The presence or absence of chylomicrons in fasting plasma was assessed both by agarose gel electrophoresis and $3 \%$ polyvinylpyrollidone (PVP) flocculation (16).

Lipoprotein phenotypes. Lipid and lipoprotein data in each survivor were analyzed by W. R. H. (with the technical assistance of Y.-L. Lee Lum) without knowledge of the survivor's genetic diagnosis. The lipoprotein phenotype of each case was assigned according to the criteria outlined in Table I. These criteria were originally developed by Fredrickson, Levy, and Lees (5) and recently modified by an advisory committee of the World Health Organization (7).

\section{RESULTS}

Variability of plasma lipid levels between initial and repeat samples. Table II contains a summary of the whole plasma and lipoprotein lipid data of the survivors of myocardial infarction in whom family studies were performed, organized according to the genetic groups delineated in the accompanying paper (10). In each of these hyperlipidemic groups, the mean level of the lipid or lipids which had been elevated in the initial plasma sample (e.g., cholesterol in familial hypercholesterolemia, triglyceride in familial hypertriglyceridemia, etc.) remained above the 99th percentile in the repeat sample (Table II). Furthermore, on an individual basis, $73 \%$ of survivors who were initially hyperlipidemic ( 295 th percentile) had lipid values in the repeat sample which 
TABLE I

Criteria for Analysis of Lipoprotein Phenotypes

\begin{tabular}{|c|c|c|c|c|}
\hline \multirow[b]{2}{*}{$\begin{array}{l}\text { Lipoprotein } \\
\text { phenotype }\end{array}$} & \multicolumn{2}{|c|}{ Plasma lipid level } & \multicolumn{2}{|c|}{ Presence of } \\
\hline & $\begin{array}{c}\text { Whole plasma } \\
\text { triglyceride } \\
\geq 95 \text { th } \\
\text { percentile* }\end{array}$ & $\begin{array}{c}\text { LDL-cholesterol } \\
\text { > upper limit } \\
\text { of normal } \neq\end{array}$ & $\begin{array}{c}\text { Fasting } \\
\text { chylomicrons§ }\end{array}$ & $\begin{array}{c}\beta \text {-migrating } \\
\text { VLDL\| }\end{array}$ \\
\hline $\mathrm{II} a$ & - & + & - & - \\
\hline $\mathrm{II} b$ & + & + & - & - \\
\hline IIIT & + or - & + or - & + or - & + \\
\hline IV & + & - & - & - \\
\hline V & + & - & + & - \\
\hline
\end{tabular}

* Based on age and sex-adjusted data from 950 control subjects (11). $\ddagger$ Based on age-related 95 th percentile values estimated by Fredrickson and Levy (9).

$\S$ By PVP flocculation and agarose gel electrophoresis.

I| By agarose gel electrophoresis (see footnote 3).

I Lipid composition of isolated VLDL (and of fasting chylomicrons, when present) and electrophoretic mobility of lipoproteins in whole plasma used as confirmatory diagnostic criteria for the type III pattern but by themselves were not considered sufficient for its assignment.

equalled or exceeded the 95th percentile. ${ }^{2}$ This proportion of survivors who remained hyperlipidemic varied among the different disorders: familial hypercholesterolemia, 93\% ; familial hypertriglyceridemia, 78\%, familial combined hyperlipidemia, 74\%; polygenic hypercholesterolemia, $75 \%$; and sporadic hypertriglyceridemia, $61 \%$. Of the 16 survivors with monogenic hyperlipidemia whose repeat lipid levels fell below the 95th percentile (including 1 with familial hypercholesterolemia, 8 with familial hypertriglyceridemia, and 7 with familial combined hyperlipidemia), 7 were taking clofibrate at the time of retesting (Tables II and III). Although the reason for the decrease in lipid levels in the other nine survivors was not clear, their repeat values tended to fall in the range between the 75th and 94th percentiles. Of the 19 survivors with either polygenic or sporadic disorders who were normolipidemic when retested, none was taking clofibrate at the time of the repeat sample (Tables II and III). Thus, untreated subjects with monogenic disorders appeared to remain hyperlipidemic on resampling to a more consistent degree than did those with polygenic and sporadic disorders.

The mean levels for both cholesterol and triglyceride among the 27 normolipidemic survivors who were tested on two occasions remained remarkably constant (Table II). Only one of these survivors had a repeat lipid value which exceeded the 95th percentile.

Between the time of the initial and repeat samples, the mean change in weight for survivors in all genetically

${ }^{2}$ The complete data from which the mean values of Table II were based have been deposited with the National Auxiliary Publication (ID no. 02056). defined groups was small and was not significantly different among normolipidemic survivors $(-0.49 \mathrm{~kg})$, those with monogenic forms of hyperlipidemia ( -0.32 $\mathrm{kg}$ ), and those with polygenic and sporadic disorders $(-0.46 \mathrm{~kg})$.

Relationship between levels of cholesterol in whole plasma and $L D L$. A major rationale for the quantitative approach to the system of lipoprotein phenotypes has been the possibility that the variable contributions to the total plasma cholesterol level from VLDL and HDL may obscure a high concentration of LDL-cholesterol and thereby mask the presence of the type II $a$ and II $b$ patterns (17). To test this relationship directly, levels of whole plasma cholesterol in the repeat samples were plotted against LDLcholesterol measured in the same samples. Statistically significant correlations $(P<0.001)$ were noted for each of the genetically defined groups: familial hypercholesterolemia, $r=+0.921$; familial hypertriglyceridemia, $r=+0.800$; familial combined hyperlipidemia, $r=+$ 0.869 ; polygenic hypercholesterolemia, $r=+0.759$; sporadic hypertriglyceridemia, $r=+0.690$; and normolipidemic survivors, $r=+0.905$. The correlation coefficient of the three monogenic groups considered together (Fig. 1A) $(r=+0.918)$ was significantly higher than that for the polygenic and sporadic groups (Fig. 1B) $(r=+0.726)(P<0.02)$. Thus, it appeared that whole plasma cholesterol closely reflected LDL-cholesterol concentrations in most subjects, particularly those with the monogenic disorders: However, in a small number of individual cases, the LDL-cholesterol level did exceed the upper limit of normal despite a whole plasma cholesterol 
TABLE II

Summary of Clinical and Biochemical Data

\begin{tabular}{|c|c|c|c|c|c|c|c|c|c|}
\hline \multirow[b]{2}{*}{$\begin{array}{c}\text { Disorder } \\
\text { (No. of subjects) }\end{array}$} & \multirow[b]{2}{*}{ Sex } & \multirow[b]{2}{*}{ Age } & \multirow[b]{2}{*}{ Height } & \multicolumn{6}{|c|}{ Initial sample } \\
\hline & & & & Weight & $\begin{array}{l}\text { Ideal } \\
\text { body } \\
\text { weight }\end{array}$ & Glucose§ & $\begin{array}{l}\text { Uric } \\
\text { acid\& }\end{array}$ & Cholesterol\| & Triglyceride \\
\hline & $\mathbf{M} / \mathbf{F}$ & $y r$ & $\mathrm{~cm}$ & kg & $\%$ & $\mathrm{mg} / 100 \mathrm{ml}$ & $m g / 100 \mathrm{ml}$ & $\mathrm{mg} / 100 \mathrm{ml}$ & $\mathrm{mg} / 100 \mathrm{ml}$ \\
\hline $\begin{array}{l}\text { Familial } \\
\text { hypercholesterolemia }\end{array}$ & & & & & & & & & \\
\hline $\begin{array}{l}(16) \\
\text { Familial } \\
\text { hypertriglyceridemia }\end{array}$ & $13 / 3$ & $48 \pm 10$ & $172 \pm 10$ & $74.3 \pm 18.6$ & $111 \pm 18$ & $88 \pm 16$ & $5.8 \pm 1.4$ & $353 \pm 62$ & $129 \pm 62$ \\
\hline (23) & $18 / 5$ & $52 \pm 10$ & $172 \pm 9$ & $77.1 \pm 14.0$ & $118 \pm 13$ & $103 \pm 40$ & $6.3 \pm 1.2$ & $241 \pm 46$ & $266 \pm 167$ \\
\hline $\begin{array}{l}\text { Familial combined } \\
\text { hyperlipidemia } \\
\text { (47) }\end{array}$ & $36 / 11$ & $51 \pm 9$ & $173 \pm 9$ & $75.6 \pm 12.8$ & $115 \pm 16$ & $97 \pm 40$ & $6.2 \pm 1.4$ & $298 \pm 52$ & $240 \pm 116$ \\
\hline $\begin{array}{l}\text { Polygenic } \\
\text { hypercholesterolemia } \\
\text { (28) }\end{array}$ & $22 / 6$ & $53 \pm 10$ & $174 \pm 8$ & $78.8 \pm 13.4$ & $118 \pm 13$ & $96 \pm 33$ & $6.3 \pm 1.7$ & $307 \pm 34$ & $184 \pm 84$ \\
\hline $\begin{array}{l}\text { Sporadic } \\
\text { hypertriglyceridemia } \\
(31)\end{array}$ & $21 / 10$ & $55 \pm 12$ & $173 \pm 10$ & $75.4 \pm 13.0$ & $115 \pm 13$ & $92 \pm 28$ & $6.4 \pm 1.2$ & $233 \pm 31$ & $243 \pm 102$ \\
\hline $\begin{array}{l}\text { Type III } \\
\text { hyperlipidemia } \\
\text { (4) }\end{array}$ & $3 / 1$ & $61 \pm 12$ & $171 \pm 8$ & $70.1 \pm 12.3$ & $109 \pm 9$ & $76 \pm 23$ & $5.1 \pm 1.3$ & $238 \pm 53$ & $234 \pm 52$ \\
\hline $\begin{array}{l}\text { Normolipidemic } \\
\text { survivors } \\
(27)\end{array}$ & $16 / 11$ & $44 \pm 10$ & $173 \pm 11$ & $70.8 \pm 13.4$ & $110 \pm 13$ & $84 \pm 13$ & $5.5 \pm 1.2$ & $226 \pm 30$ & $97 \pm 42$ \\
\hline
\end{tabular}

* The data for each disorder are expressed as mean values $\pm 1 \mathrm{SD}$. The complete data on individual subjects from which these mean values were derived have been deposited with the National Auxiliary Publications Service (ID no. 02056).

I Ideal body weight was determined by criteria of the Metropolitan Life Insurance Company as described in the accompanying paper (11).

$\$$ Glucose and uric acid levels were measured on fasting plasma samples as described in the accompanying paper (11).

II Unadjusted for age and sex.

I $\Delta$ weight $=$ weight at time of repeat sample-weight at time of initial sample.

concentration which lay below the 95th percentile. This phenomenon occurred in only one subject with familial hypertriglyceridemia, eight with familial combined hyperlipidemia, two with polygenic hypercholesterolemia, and four with sporadic hypertriglyceridemia. It was also seen in three normolipidemic survivors (Table II).

The relationship between the content of triglyceride in whole plasma and in VLDL was also very close, with a correlation coefficient greater than +0.959 in all genetically defined groups including the normolipidemic survivors. Thus, the simple determination of whole plasma triglyceride accurately reflected its concentration in VLDL in all survivors.

Type III lipoprotein patterns in survivors. Unequivocal evidence for the type III pattern was present in only one survivor. This individual (no. 115, Table II) manifested the typical features of this disorder (9): hypercholesterolemia; hypertriglyceridemia; $\beta$-migrating VLDL ; ${ }^{3}$ cholesterol-rich VLDL (cholesterol/triglycer-

${ }^{3}$ When the electrophoretic mobility of isolated VLDL from subjects with the typical type III disorder (identified in a previous study [18]) was compared on paper and agarose gel, $\beta$-VLDL migrated slightly faster in agarose to a "slow" pre- $\beta$-position. However, in the present study such mobility has been designated as ' $\beta$ " to permit comparison

\section{2}

ide $=0.474)(18)$; a low level of LDL-cholesterol; and cholesterol-rich fasting chylomicrons isolated by PVP flocculation (cholesterol/triglyceride $=0.41$ ) (19). In addition, polyacrylamide gel electrophoresis of his whole plasma revealed the characteristic trail of lipoproteins migrating in a zone intermediate between normal VLDL and normal LDL, both of which were barely detectable in this subject (Fig. 2). A type III pattern was also present in the $19 \mathrm{yr}$ old son of this survivor (Fig. 2).

In addition to this case, four other hyperlipidemic survivors were judged to have $\beta$-migrating VLDL, ${ }^{8}$ but in none was the evidence for the type III pattern completely convincing in either the survivors themselves or in their hyperlipidemic relatives. Survivors 293 and 496 had a faint band of $\beta$-migrating VLDL, but in each case the predominant VLDL on agarose gel had a normal, pre- $\beta$-mobility. Although polyacrylamide gel electrophoresis in these cases (Fig. 2) revealed lipoproteins migrating as a trail intermediate in position between normal VLDL and LDL, it also showed a more dense band in the position of LDL than has been noted in more clear-cut examples of the type III pattern. In addition,

with other reports in which paper electrophoresis had been employed (9). 
on 176 Survivors of Myocardial Infarction*

\begin{tabular}{|c|c|c|c|c|c|c|c|c|}
\hline \multirow{3}{*}{$\begin{array}{l}\text { Interval } \\
\text { between } \\
\text { samples }\end{array}$} & \multicolumn{8}{|c|}{ Repeat sample } \\
\hline & \multirow[b]{2}{*}{$\Delta$ Weight I } & \multirow[b]{2}{*}{ Cholesterol\| } & \multirow[b]{2}{*}{ Triglyceride\| } & \multicolumn{3}{|c|}{ VLDL } & \multirow{2}{*}{$\begin{array}{c}\text { LDL } \\
\text { cholesterol }\end{array}$} & \multirow{2}{*}{$\begin{array}{c}\text { HDL } \\
\text { cholesterol }\end{array}$} \\
\hline & & & & Cholesterol & Triglyceride & $\mathrm{C} / \mathrm{T}$ & & \\
\hline days & $k g$ & $\mathrm{mg} / 100 \mathrm{ml}$ & $m g / 100 m l$ & $m g / 100 \mathrm{ml}$ & $m g / 100 \mathrm{ml}$ & & $\mathrm{mg} / 100 \mathrm{ml}$ & $\mathrm{mg} / 100 \mathrm{ml}$ \\
\hline $126 \pm 45$ & $-0.56 \pm 2.9$ & $349 \pm 77$ & $167 \pm 91$ & $38 \pm 29$ & $99 \pm 71$ & $0.36 \pm 0.09$ & $261 \pm 61$ & $43 \pm 21$ \\
\hline $115 \pm 72$ & $-0.22 \pm 3.6$ & $240 \pm 51$ & $240 \pm 252$ & $50 \pm 52$ & $172 \pm 213$ & $0.32 \pm 0.09$ & $155 \pm 36$ & $33 \pm 8$ \\
\hline $100 \pm 50$ & $-0.31 \pm 2.7$ & $281 \pm 45$ & $215 \pm 174$ & $41 \pm 33$ & $150 \pm 95$ & $0.31 \pm 0.23$ & $197 \pm 50$ & $42 \pm 16$ \\
\hline $93 \pm 37$ & $-0.25 \pm 2.6$ & $288 \pm 36$ & $169 \pm 86$ & $33 \pm 20$ & $106 \pm 73$ & $0.32 \pm 0.10$ & $206 \pm 43$ & $47 \pm 28$ \\
\hline $96 \pm 38$ & $-0.64 \pm 2.4$ & $249 \pm 34$ & $214 \pm 87$ & $45 \pm 27$ & $144 \pm 93$ & $0.35 \pm 0.14$ & $164 \pm 51$ & $37 \pm 14$ \\
\hline $86 \pm 54$ & $-0.33 \pm 2.2$ & $264 \pm 56$ & $279 \pm 146$ & $93 \pm 69$ & $202 \pm 143$ & $0.45 \pm 0.01$ & $134 \pm 60$ & $36 \pm 13$ \\
\hline $197 \pm 92$ & $-0.49 \pm 3.6$ & $226 \pm 35$ & $96 \pm 38$ & $17 \pm 8$ & $58 \pm 29$ & $0.32 \pm 0.12$ & $162 \pm 32$ & $45 \pm 12$ \\
\hline
\end{tabular}

neither of these subjects had fasting chylomicrons. Their VLDL cholesterol/triglyceride ratios lay between the ranges observed in groups of subjects with well-defined type III patterns $(20.48)$ and type IV patterns $(\leq 0.42)$ as determined in a previous study (18). The other two survivors (108 and 366 ) were of special interest because they manifested both $\beta$-migrating VLDL and high levels of LDL-cholesterol, a combination hitherto reported only in a patient with severe concurrent hypothyroidism (20) and in a single subject with a familial form of hypercholesterolemia (21). Neither of these two cases had clinical evidence of hypothyroidism; one of them (108) was the proband of a pedigree that met the diagnostic criteria for familial hypercholesterolemia (10). In both cases polyacrylamide gel electrophoresis revealed only faint bands in the position of normal LDL (Fig. 2). Of the two cases, only no. 366 (VLDL cholesterol/triglyceride ratio of 0.45 ) was grouped with the type III subjects in Table II. Survivor 108 was included among those with familial hypercholesterolemia on the basis of the family data and the high-normal VLDL cholesterol/ triglyceride ratio of 0.40 .

These data indicate that the subjects with $\beta$-VLDL in this study comprised a heterogeneous group in terms of both genetic and lipoprotein characteristics. Moreover, if an elevated VLDL cholesterol/triglyceride ratio had been used as the sole criterion for the diagnosis of the type III pattern, the number of apparent type III subjects would have been increased by four (cases 5, 158, 316, and 339). None of these four subjects, however, showed clear-cut electrophoretic evidence of $\beta$-VLDL. Thus, neither $\beta$-VLDL nor cholesterol-rich VLDL proved to be a specific marker for a distinct genetic disorder. Because of these diagnostic uncertainties, the frequency of the type III pattern among the 500 survivors of myocardial infarction identified in the accompanying paper (11) could only be roughly estimated at between 0.2 and $1.0 \%$.

Relationship between genetic classification and lipoprotein phenotypes. Table III shows the distribution of lipoprotein phenotypes in 133 survivors with five different genetically defined forms of hyperlipidemia (10). As indicated in the table, no lipoprotein pattern was specific for any one genetically defined lipid disorder; conversely, no genetic disorder was specified by any single lipoprotein pattern. However, there were several trends in the relationship between genetic classification and lipoprotein phenotypes. First, the most frequent lipoprotein phenotypes in survivors with the familial hyper-

Hyperlipidemia in Coronary Heart Disease 
TABLE III

Relationship between Genetic Classification and Lipoprotein Phenotypes in Hyperlipidemic Survivors of Myocardial Infarction

\begin{tabular}{|c|c|c|c|c|c|c|}
\hline \multirow[b]{2}{*}{ Genetic disorder } & \multirow{2}{*}{$\begin{array}{c}\text { No. } \\
\text { of } \\
\text { subjects }\end{array}$} & \multicolumn{5}{|c|}{ Lipoprotein phenotype } \\
\hline & & Normal & $\mathrm{II} a$ & $I \mathrm{I} b$ & IV & 1 \\
\hline \multicolumn{7}{|l|}{ Monogenic } \\
\hline Familial & & & & & & \\
\hline $\begin{array}{l}\text { hypercho- } \\
\text { lesterolemia }\end{array}$ & 14 & $1^{*}$ & 7 & 4 & $2 \ddagger$ & \\
\hline Familial & & & & & & \\
\hline $\begin{array}{l}\text { hypertri- } \\
\text { glyceridemia }\end{array}$ & 22 & $8 \S$ & 0 & $2 \|$ & 11 & \\
\hline $\begin{array}{c}\text { Familial combined } \\
\text { hyperlipidemia }\end{array}$ & 41 & 79 & $13^{* *}$ & 10 & 10 & \\
\hline \multicolumn{7}{|l|}{ Polygenic } \\
\hline $\begin{array}{l}\text { Hyperchol- } \\
\text { esterolemia }\end{array}$ & 25 & 9 & 9 & 2 & 5 & \\
\hline Sporadic & & & & & & \\
\hline $\begin{array}{l}\text { Hypertri- } \\
\text { glyceridemia }\end{array}$ & 31 & 10 & $4 \ddagger t$ & 2 & 14 & \\
\hline
\end{tabular}

* Taking clofibrate at the time of the repeat sample.

$\ddagger$ One of these subjects was under treatment for acute hyperthyroidism with ${ }^{131}$ I and prednisone at the time of the repeat sample.

$\S$ Four were taking clofibrate.

\| One was taking clofibrate.

\ Two were taking clofibrate.

** Four were taking clofibrate.

$\ddagger \ddagger$ One was taking clofibrate.

cholesterolemic disorder were the type II $a$ and type II $b$ patterns. Second, the most characteristic lipoprotein phenotype in survivors with the familial hypertriglyceridemic disorder was the type IV pattern. Third, survivors with the familial combined hyperlipidemic disorder manifested the widest variety of lipoprotein patterns: types II $a, \mathrm{II} b, \mathrm{IV}$, and V. A similar distribution of phenotypes was observed among the affected relatives of a large kindred with familial combined hyperlipidemia (see the accompanying paper [10]).

Since the LDL-cholesterol level was an important determinant of an individual's lipoprotein phenotype, it was important to compare the distribution of the LDLcholesterol levels of hyperlipidemic and normolipidemic survivors grouped according to their genetic diagnoses (Fig. 3). Although the individual values showed a significant variation within each genetic group and thus many instances of overlap occurred between groups, the mean LDL-cholesterol level did tend to discriminate statistically $(P<0.005)$ among the three monogenic disorders. LDL-cholesterol levels were highest in familial hypercholesterolemia $(261 \pm 61 \mathrm{mg} / 100 \mathrm{ml}$ [mean $\pm S D]$ ), intermediate in familial combined hyperlipi-
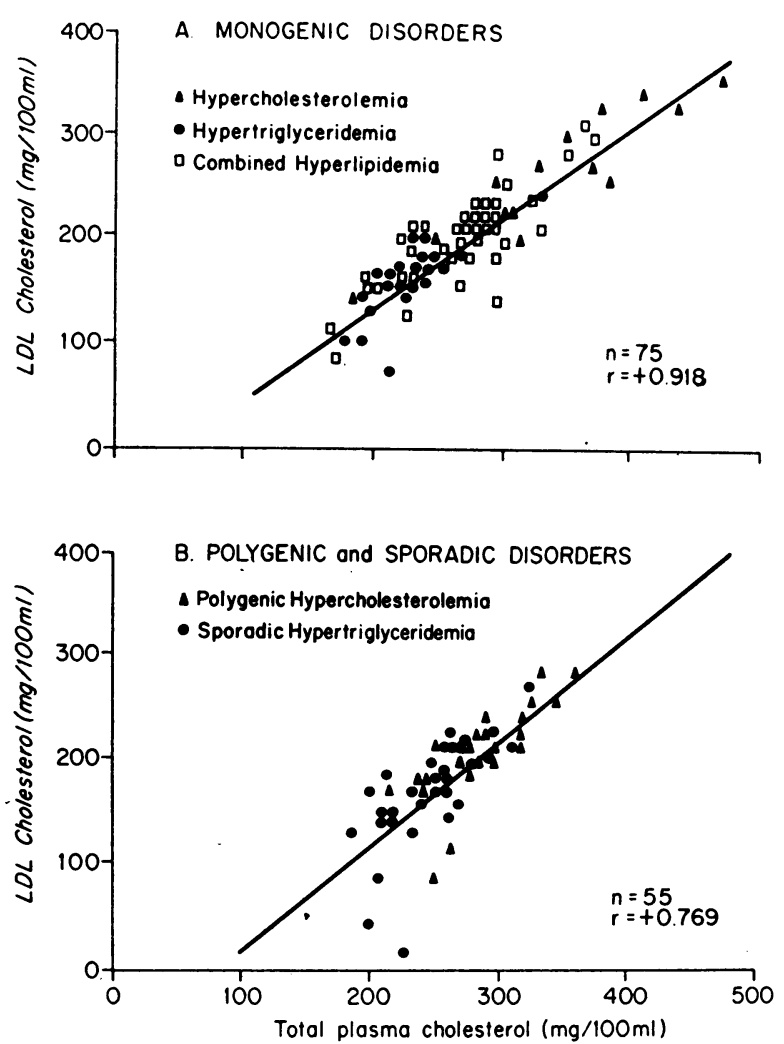

Figure 1 (A) Relationship between total plasma cholesterol and low density lipoprotein (LDL)-cholesterol levels in 75 survivors with monogenic lipid disorders. Included in this group of survivors were 14 with familial hypercholesterolemia, 21 with familial hypertriglyceridemia, and 40 with familial combined hyperlipidemia. Two survivors with hypertriglyceridemia associated with hyperchylomicronemia were omitted. Both the whole plasma cholesterol and the LDL-cholesterol levels were determined on the same repeat plasma sample for each survivor. (B) Relation between whole plasma cholesterol and LDL-cholesterol levels in 54 survivors with polygenic hypercholesterolemia $(n=25)$ and sporadic hypertriglyceridemia $(n=29)$. One survivor with hypertriglyceridemia associated with hyperchylomicronemia was omitted. Both the whole plasma cholesterol and the LDLcholesterol levels were determined on the same plasma sample for each survivor.

demia $(197 \pm 50)$, and lowest in familial hypertriglyceridemia $(155 \pm 36)$. However, the mean LDL-cholesterol level was not significantly different between the polygenic hypercholesterolemic group $(206 \pm 43)$ and the combined hyperlipidemic group $(197 \pm 50)$, nor was it different between the familial $(155 \pm 36)$ and the sporadic $(164 \pm 51)$ hypertriglyceridemic groups. It was of note that the mean LDL-cholesterol level of the familial combined hyperlipidemic group was very close to the suggested upper limit of normal for middle-aged subjects. Thus, depending on whether one's value fell above or below this mean, an individual with familial combined hy-

\section{4 \\ Hazzard, Goldstein, Schrott, Motulsky, and Bierman}




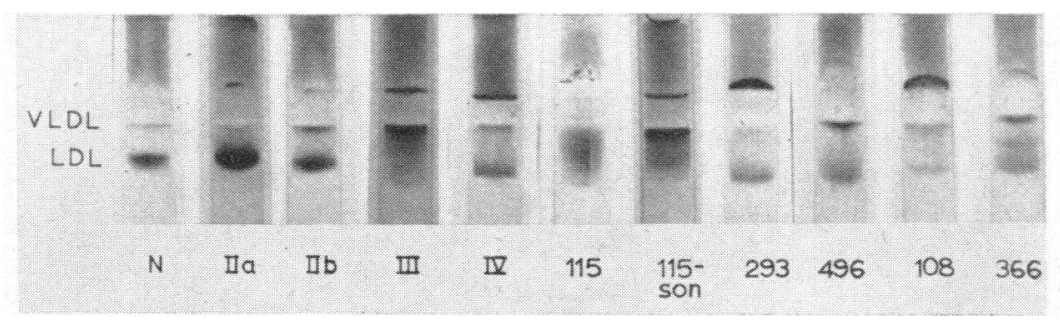

7 i] Figure 2 Whole plasma lipoprotein electrophoretic patterns in polyacrylamide gel. The positions of normal VLDL and LDL are indicated along the left margin. The first five patterns were observed in subjects not included in this study and are typical for normal (N) and II $a$, II $b$, III, and IV lipoprotein phenotypes, respectively. The arabic numerals designate patterns in subjects in this study according to their identification numbers (see text and Table III). 115 -son is the son of case 115 .

perlipidemia might show a type II $a$, II $b$, or IV lipoprotein pattern.

\section{DISCUSSION}

Lipoprotein phenotypes are now extensively used to diagnose and classify the familial hyperlipidemias (7). Despite this widespread acceptance and application in clinical medicine, no full evaluation of this system, based on family studies, has been published to date. Consequently, several questions have remained unanswered. For example, does lipoprotein quantification and typing provide important information beyond that obtained from the simple measurement of whole plasma lipid levels? Does each of the lipoprotein patterns reflect a different and specific genetic disorder? Do the different lipoprotein types relate to the familial distribution, pathophysiology, prognosis, and therapy of hyperlipidemia in the individual patient? Since in the present study a genetic diagnosis of a large number of hyperlipidemic survivors of myocardial infarction was determined, analysis of their lipoprotein patterns provided the opportunity to approach several of these questions.

A possible limitation in the design of this study was the determination of the lipoprotein phenotype of each survivor on a repeat rather than on the initial plasma sample. However, since $88 \%$ of the 176 survivors who underwent a family study (10) were available for repeat sample, the ascertainment bias which might have been associated with obtaining repeat samples was minimized. Furthermore, considering the variety of metabolic stresses experienced by the survivors in the interval between the two samples (e.g., alterations in diet and drug therapy, recurrence of chest pain or hospitalization), their lipid levels proved to be remarkably stable.

Perhaps the most important result of the present study was the demonstration that in the individual hyperlipidemic survivor there was no consistent relationship between lipoprotein phenotype and genetic disorder. Several factors appeared to contribute to this lack of association: First, lipoprotein phenotypes were assigned using arbitrary 95th percentile cut-off limits for both plasma triglyceride and LDL-cholesterol. Consequently, it was not uncommon for individual survivors with genetic evidence for a familial form of hyperlipidemia to have a normal phenotype. Second, lipoprotein phenotypes failed to distinguish among the monogenic, polygenic, and sporadic forms of hyperlipidemia. Thus, determination of the lipoprotein pattern in the individual survivor did not predict whether family studies would show additional affected relatives. Third, lipoprotein phenotypes failed to separate the three monogenic disorders in the individual case because of significant overlap in LDL-cholesterol values. Fourth, since the various lipoprotein patterns differed quantitatively rather than qualitatively, a small difference in the level of either LDL-cholesterol or whole plasma triglyceride resulted in the assignment of different lipoprotein phenotypes to individuals in the

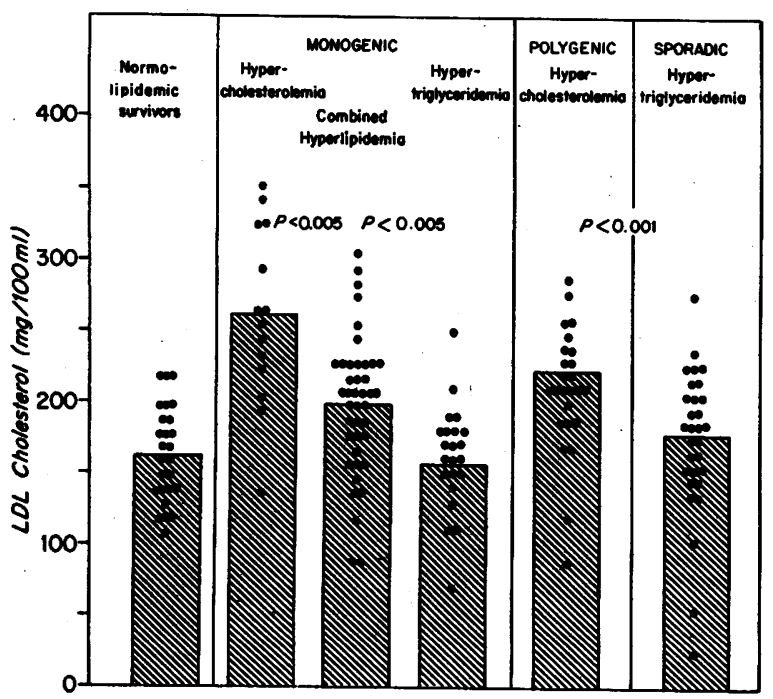

FIGURE 3 LDL-cholesterol levels in survivors of myocardial infarction grouped according to their genetic diagnosis. The LDL-cholesterol measurements were carried out on each survivor's repeat plasma sample.

Hyperlipidemia in Coronary Heart Disease 
same genetic group. This occurred most frequently in those with familial combined hyperlipidemia, who as a group manifested four different lipoprotein patterns: types II $a$, II $b$, IV, and V. Fifth, heterogeneity in both genetic and lipoprotein characteristics was also evident among survivors with the type III pattern. When the type III pattern was defined by the single criterion of the presence of $\beta$-migrating VLDL, the group of subjects so identified varied in the lipid composition of their VLDL, the level of LDL-cholesterol, the electrophoretic migration of whole plasma lipoproteins on polyacrylamide gel, the presence of cholesterol-rich fasting chylomicrons, and in their familial lipid distributions. From these data, it would appear that $\beta$-VLDL may not be a specific marker for a single and distinct lipid disorder, as has been previously suggested (9).

These studies have also served to justify the use of whole plasma lipid levels for genetic and epidemiological investigations of hyperlipidemia, since excellent correlations were observed between whole plasma and lipoprotein lipid levels in both hyperlipidemic and normolipidemic survivors. In addition, diagnosis on the basis of the family patterns derived from quantitative analysis of whole plasma lipid levels in relatives avoids the misclassification of the index case resulting from the use of arbitrary cut-off limits inherent in determining the lipoprotein phenotype. And finally, unlike the methods for analysis of lipoprotein phenotypes which require a relatively large volume of fresh or refrigerated (unfrozen) plasma, whole plasma lipid levels can be measured in small blood samples collected in diverse geographical regions and mailed at ambient temperature to a single laboratory.

Thus the present study emphasizes that lipoprotein phenotypes are not markers in the genetic sense and as such cannot be equated with specific genetic disorders. This conclusion is reinforced by three lines of previous evidence: first, variation in the lipoprotein phenotype of a given individual often occurs under varying physiological and pharmacological conditions (e.g., conversion of a type $\mathrm{V}$ to a type IV pattern with caloric deprivation [22]) ; second, different lipoprotein phenotypes often occur in several members of a single family (e.g., III and IV, I and V, II $a$ and II $b$, II and IV, and IV and $\mathrm{V}$ [9]) ; and finally, acquired (i.e., secondary) forms of each abnormal lipoprotein pattern have been reported and cannot be distinguished by lipoprotein phenotype from genetic and sporadic forms (9).

Until the fundamental biochemical defects are identified, quantitative analysis of the variation in lipid levels in relatives of hyperlipidemic probands (10) would seem to provide a more meaningful method for genetic classification of hyperlipidemia than analysis of the lipoprotein phenotype of the individual hyperlipidemic subject. By providing patients with a genetic diagnosis, this technique offers a promising approach in the design of studies aimed at determining the underlying metabolic abnormality characteristic of each disorder.

\section{ACKNOWLEDGMENTS}

We are indebted to the following colleagues for their able assistance in these studies: Stanley Albert for computer programming; Susan Mar, Kathryn Bakeman, and Martha Salsburg for clerical help; Y.-L. Lee Lum, Karen Grams, Marilyn Vogel, and Martha Kimura for lipid analyses.

This work was supported by U. S. Public Health Service Grants GM 15253, AM 06670, and HD 04872; by a grant from the Washington State Heart Association; by National Institutes of Health contract 71-2157; and by the Veterans Administration.

\section{REFERENCES}

1. Goldstein, J. L., W. R. Hazzard, H. G. Schrott, E. L. Bierman, and A. G. Motulsky. 1972. Genetics of hyperlipidemia in coronary heart disease. Trans. Assoc. Am. Physicians Phila. 85: 120.

2. Gofman, J. W., L. Rubin, J. P. McGinley, and H. B. Jones. 1954. Hyperlipoproteinemia. Am. J. Med. 17: 514.

3. Thannhauser, S. J. 1958. Lipidoses: Diseases of the Intracellular Lipid Metabolism. Grune \& Stratton, Inc., New York. 3rd edition. 78.

4. Ahrens, E. H., Jr., J. Hirsch, K. Oette, J. W. Farquhar, and Y. Stein. 1961. Carbohydrate-induced lipemia. Trans. Assoc. Am. Physicians Phila. 74: 134.

5. Fredrickson, D. S., R. I. Levy, and R. S. Lees. 1967. Fat transport in lipoproteins-an integrated approach to mechanisms and disorders. N. Engl. J. Med. 276: 32, 94, $148,215,273$.

6. Havel, R. J. 1970. Typing of hyperlipoproteinemias. Atherosclerosis. $11: 3$.

7. Beaumont, J. L., L. A. Carlson, G. R. Cooper, Z. Fejfar, D. S. Fredrickson, and T. Strasser. 1970. Classification of hyperlipidemias and hyperlipoproteinemias. Bull.W.H. O. 43: 891 .

8. Harlan, W. R., P. S. Winesett, and A. J. Wasserman. 1967. Tissue lipoprotein lipase in normal individuals and individuals with exogenous hypertriglyceridemia and the relationship of this enzyme to assimilation of fat. $J$. Clin. Invest. 46: 239.

9. Fredrickson, D. S., and R. I. Levy. 1972. Familial hyperlipoproteinemia. In The Metabolic Basis of Inherited Disease. J. B. Stanbury. J. B. Wyngaarden, and D. S. Fredrickson. McGraw-Hill Book Company, New York. 3rd edition. 545.

10. Goldstein, J. L., H. G. Schrott, W. R. Hazzard, E. L. Bierman, and A. G. Motulsky. 1973. Hyperlipidemia in coronary heart disease. II. Genetic analysis of lipid levels in 176 families and delineation of a new inherited disorder, combined hyperlipidemia. J. Clin. Invest. 52: 1544.

11. Goldstein, J. L., W. R. Hazzard, H. G. Schrott, E. L. Bierman, and A. G. Motulsky. 1973. Hyperlipidemia in coronary heart disease. I. Lipid levels in 500 survivors of myocardial infarction. J. Clin. Invest. 52: 1533. 
12. Quarfordt, S. H., F. Boston, and H. Hilderman. 1971. Transfer of triglyceride between isolated human lipoproteins. Biochim. Biophys. Acta. 231: 290.

13. Burstein, M., and J. Samaille. 1960. Sur un dosage rapide du cholesterol lié aux alpha et aux beta-lipoproteines du sérum. Clin. Chim. Acta. 5: 609.

14. Noble, R. P. 1968. Electrophoretic separation of plasma lipoproteins in agarose gel. J. Lipid Res. 9: 693.

15. Davis, B. J. 1959. Disc electrophoresis. II. Method and application to human serum proteins. Ann. N. Y. Acad. Sci. 121: 404.

16. O'Hara. D. D., D. Porte, Jr., and R. H. Williams. 1966. Use of constant composition polyvinylpyrrolidone columns to study the interaction of fat particles with plasma. J. Lipid Res. $7: 264$.

17. Rifkind, B. 1970. Typing of hyperlipoproteinaemias. Atherosclerosis. $11: 545$.

18. Hazzard, W. R., D. Porte, Jr., and E. L. Bierman.
1972. Abnormal lipid composition of very low density lipoproteins in the diagnosis of broad-beta disease (Type III hyperlipoproteinemia). Metab. (Clin. Exp.). 21: 1009.

19. Hazzard, W. R., D. Porte, Jr., and E. L. Bierman. 1970 Abnormal lipid composition of chylomicrons in broad$\beta$ disease (type III hyperlipoproteinemia). J. Clin. Invest. 49 : 1853.

20. Hazzard, W. R., and E. L. Bierman. 1972. Aggravation of broad-beta disease (Type III hyperlipoproteinemia) by hypothyroidism. Arch. Intern. Med. 130: 822.

21. Lasser, N. L., and S. Katz. 1972. The occurrence of type II and type III hyperlipoproteinemia in a single kindred. Clin. Res. 20: 549. (Abstr.)

22. Brunzell, J. D., D. Porte, Jr., and E. L. Bierman. 1971 Evidence for a common saturable removal system for dietary and endogenous triglyceride in man. J. Clin. Invest. 50: 15a. (Abstr.) 